\title{
The Pontryagin maximum principle as a necessary and sufficient optimality condition in a variable structure optimal control problem
}

\author{
K.B. Mansimov ${ }^{\mathrm{a}, \mathrm{b}^{*}}$, V.G. Rzayeva ${ }^{\mathrm{c}}$ \\ ${ }^{a}$ Institute of Control Systems of Azerbaijan National Academy of Sciences, Baku, Azerbaijan \\ ${ }^{b}$ Baku State University, Baku, Azerbaijan \\ ${ }^{c}$ Sumgayit State University, Sumgayit, Azerbaijan
}

\begin{tabular}{|c|c|}
\hline A R T I C L E I N F O & A B S T RA C T \\
\hline $\begin{array}{l}\text { Article history: } \\
\text { Received 12.02.2021 } \\
\text { Received in revised form } 23.02 .2021 \\
\text { Accepted } 11.03 .2021 \\
\text { Available online } 29.12 .2021\end{array}$ & $\begin{array}{l}\text { The authors consider one optimal control problem with a variable } \\
\text { structure, described in various domains by a Goursat-Darboux } \\
\text { system and a two-dimensional Volterra integral equation. Using } \\
\text { one version of the method of increments, a necessary and sufficient } \\
\text { condition for optimality is proved in the form of the Pontryagin }\end{array}$ \\
\hline $\begin{array}{l}\text { Keywords: } \\
\text { Pontryagin maximum principle } \\
\text { Necessary and sufficient optimality } \\
\text { condition } \\
\text { Increment formula } \\
\text { Volterra integral equation } \\
\text { System of hyperbolic equations } \\
\text { Linear equation }\end{array}$ & \\
\hline
\end{tabular}

\section{Introduction}

By now, the theory of necessary optimality conditions for optimal control problems described by partial differential equations of hyperbolic type with Goursat boundary conditions or integral equations has been studied quite comprehensively by various authors. A review of the relevant studied is available, for instance, in [1-3] and others.

In this article, we investigate one optimal control problem described in various phase spaces by a linear Goursat-Darboux system and a two-dimensional linear integral equation of the Volterra type. The necessary and sufficient optimality condition is proved by the increment method.

\section{Problem statement}

Suppose that $R^{r}$ and $R^{q}$ are linear $r$-and $q$-dimensional spaces, respectively, $D_{i}=\left[t_{0}, t_{i}\right] \times$ $\left[x_{0}, x_{1}\right], i=1,2$, are specified rectangles, $U \in R^{r}, V \in R^{q}$ are non-empty and bounded sets.

Consider the problem of the minimum of the terminal type of the functional

$$
S(u, v)=c^{\prime} z\left(t_{1}, x_{1}\right)+d^{\prime} y\left(t_{2}, x_{1}\right),
$$

subject to the constraints

\footnotetext{
${ }^{*}$ Corresponding author.

E-mail addresses: kamilbmansimov@gmail.com (K.B. Mansimov), kmansimov@mail.ru (V.G. Rzayeva). 


$$
\begin{gathered}
u(t, x) \in U,(t, x) \in D_{1}, \\
v(t, x) \in V,(t, x) \in D_{2}, \\
z_{t x}(t, x)=A(t, x) z+B(t, x) z_{t}+C(t, x) z_{x}+f(t, x, u), \\
z\left(t_{0}, x\right)=a(x), x \in\left[x_{0}, x_{1}\right], \\
z\left(t, x_{0}\right)=b(t), t \in\left[t_{0}, t_{1}\right], \\
a\left(x_{0}\right)=b\left(t_{0}\right), \\
y(t, x)=\int_{t_{1}}^{t} \int_{x_{0}}^{x}[D(t, x, \tau, s) y(\tau, s)+g(t, x, \tau, s, v(\tau, s))] d s d \tau+E(x) z\left(t_{1}, x\right) .
\end{gathered}
$$

Here, $z(t, x) \in R^{n}, y(t, x) \in R^{m}$ are system state vectors, $c, d$ are specified constant vectors, $u(t, x)(v(t, x))$ is the measurable and bounded $r(q)$-dimensional vector function of control actions, $A(t, x), B(t, x), C(t, x)$ are specified $(n \times n)$ measurable and bounded matrix functions, $D(t, x, \tau, s)$ is a specified $(m \times m)$ measurable and bounded matrix function, $f(t, x, u)(g(t, x, \tau, s, v))$ is a specified, $n(m)$-dimensional vector function continuous in the set of variables, $a(x), b(t)$ are specified Lipschitz vector functions, $E(x)$ is a specified continuous $(m \times n)$ - matrix function.

We shall call the pair of control functions $(u(t, x), v(t, x))$ with the above properties an admissible control.

Suppose that for each given admissible control the boundary value problem (4)-(5) has a unique, absolutely continuous solution $[4,5]$, and the integral equation (6) has a unique continuous solution [6].

The admissible control $\left(u^{0}(t, x), v^{0}(t, x)\right)$ that satisfies a minimum value to functional (1) subject to constraints (2)-(6) shall be called the optimal control, and the corresponding process $\left(u^{0}(t, x), v^{0}(t, x), z^{0}(t, x), y^{0}(t, x)\right)$ shall be called the optimal process.

\section{The formula for the increment of the quality functional and the conjugate system}

Assuming that $\left(u^{0}(t, x), v^{0}(t, x), z^{0}(t, x), y^{0}(t, x)\right)$ is a fixed process, and $(\bar{u}(t, x)=$ $u^{0}(t, x)+\Delta u(t, x), \bar{v}(t, x)=v^{0}(t, x)+v(t, x), \bar{z}(t, x)=z^{0}(t, x)+\Delta z(t, x), \bar{y}(t, x)=y^{0}(t, x)+$ $\Delta y(t, x))$ are arbitrarily admissible processes, we write the increment of quality functional (1) in the following form:

$$
\begin{gathered}
\Delta S\left(u^{0}, v^{0}\right)=S(\bar{u}, \bar{v})-S\left(u^{0}, v^{0}\right)= \\
=c^{\prime} \Delta z\left(t_{1}, x_{1}\right)+d^{\prime} \Delta y\left(t_{2}, x_{1}\right),
\end{gathered}
$$

It is clear from (4)-(6) that the increment $(\Delta z(t, x), \Delta y(t, x))$ of the state $\left(z^{0}(t, x), y^{0}(t, x)\right)$ satisfies the relations

$$
\begin{gathered}
\Delta z_{t x}(t, x)=A(t, x) \Delta z(t, x)+B(t, x) \Delta z_{t}(t, x)+C(t, x) \Delta z_{x}(t, x)+ \\
+f(t, x, \bar{u}(t, x))-f\left(t, x, u^{0}(t, x)\right), \\
\Delta z\left(t_{0}, x\right)=0, x \in\left[x_{0}, x_{1}\right] \\
\Delta z\left(t, x_{0}\right)=0, t \in\left[t_{0}, t_{1}\right] \\
\Delta y(t, x)=\int_{t_{1}} \int_{x_{0}}^{x}\left[D(t, x, \tau, s) \Delta y(\tau, s)+\left(g(t, x, \tau, s, \bar{v}(\tau, s))-g\left(t, x, \tau, s, v^{0}(\tau, s)\right)\right)\right] d s d \tau+ \\
+E(x) \Delta z\left(t_{1}, x\right) .
\end{gathered}
$$


Suppose that $\psi_{1}^{0}, \psi_{2}^{0}$ are as yet arbitrary $n$ - and $m$-dimensional vector functions. Taking into account identities (8), (10), we have

$$
\begin{gathered}
\int_{t_{0}}^{t_{1}} \int_{x_{0}}^{x_{1}} \psi_{1}^{0^{\prime}}(t, x) \Delta z_{t x}(t, x) d x d t= \\
=\int_{t_{0}}^{t_{1} x_{x_{0}}} \int_{1}^{x_{1}} \psi_{1}^{0^{\prime}}(t, x)\left[A(t, x) \Delta z(t, x)+B(t, x) \Delta z_{t}(t, x)+C(t, x) \Delta z_{x}(t, x)+\right. \\
\left.+\left(f(t, x, \bar{u}(t, x))-f\left(t, x, u^{0}(t, x)\right)\right)\right] d x d t \\
=\int_{t_{1}}^{t_{2}} \int_{x_{0}}^{t_{2}} \psi_{t_{1}}^{x_{1}} \int_{x_{0}}^{0^{\prime}}(t, x)\left[\int_{t_{1}}^{t} \int_{x_{0}}^{x}[D(t, x, \tau, s) \Delta y(\tau, s)+\right. \\
\left.+\left(g(t, x, \tau, s, \bar{v}(\tau, s))-g\left(t, x, \tau, s, v^{0}(\tau, s)\right)\right)\right] d s d \tau+ \\
+\int_{t_{1}}^{t_{2}} \int_{x_{0}}^{x_{1}} \psi_{2}^{0^{\prime}}(t, x) E(x) \Delta z\left(t_{1}, x\right) d x d t .
\end{gathered}
$$

Taking into account boundary conditions (9), it is clear that

$$
\begin{gathered}
\Delta z(t, x)=\int_{t_{1}}^{t} \int_{x_{0}}^{x} \Delta z_{\tau s}(\tau, s) d s d \tau, \\
\Delta z_{t}(t, x)=\int_{x_{0}}^{x} \Delta z_{t s}(t, s) d s \\
\Delta z_{x}(t, x)=\int_{t_{0}}^{t} \Delta z_{\tau x}(\tau, x) d \tau .
\end{gathered}
$$

Further, applying the Fubini theorem (the Dirichlet formula (see, e.g., $[7,8]$ )) we obtain

$$
\begin{gathered}
\int_{t_{1}}^{t_{2}} \int_{x_{0}}^{x_{1}} \psi_{2}^{0^{\prime}}(t, x)\left[\int_{t_{1}}^{t} \int_{x_{0}}^{x}[D(t, x, \tau, s) \Delta y(\tau, s)+\right. \\
\left.+\left(g(t, x, \tau, s, \bar{v}(\tau, s))-g\left(t, x, \tau, s, v^{0}(\tau, s)\right)\right)\right] d s d \tau= \\
=\int_{t_{1}}^{t_{2}} \int_{x_{0}}^{x_{1}}\left[\int_{t}^{t_{2}} \int_{x}^{x_{1}} \psi_{2}^{0^{\prime}}(\tau, s)[D(\tau, s, t, x) \Delta y(t, x)+\right. \\
\left.+\left(g(\tau, s, t, x, \bar{v}(t, x))-g\left(\tau, s, t, x, v^{0}(t, x)\right)\right) d s d \tau\right] d x d t .
\end{gathered}
$$




$$
\begin{gathered}
\int_{t_{0}}^{t_{1}} \int_{t_{0}}^{x_{1}} \psi_{1}^{0^{\prime}}(t, x) A(t, x) \Delta z(t, x) d x d t= \\
\left.=\int_{t_{0}}^{t_{1}} \int_{t_{1}}^{x_{1}} \int_{t_{1}}^{t_{2}} \int_{x}^{x_{1}} \psi_{1}^{0^{\prime}}(\tau, s) A(\tau, s) d s d \tau\right) \Delta z_{t x}(t, x) d x d t, \\
=\int_{t_{0}}^{t_{t_{1}}} \int_{x_{0}}^{x_{1}} \psi_{x_{0}}^{x_{1}} \int_{x}^{x_{1}}(t, x) B(t, x) \Delta z_{t}(t, x) d x d t= \\
\left.\int_{t_{0}}^{t_{1}} \int_{x_{0}}^{x_{1}} \psi_{1}^{0^{\prime}}(t, s) B(t, s) d s\right) \Delta z_{t x}(t, x) d x d t, \\
=\int_{t_{0}}^{t_{1}} \int_{x_{0}}^{x_{1}}\left(\int_{t}^{t_{1}} \psi_{1}^{0^{\prime}}(\tau, x) C(\tau, x) d \tau\right) \Delta z_{t x}(t, x) d x d t .
\end{gathered}
$$

From (13) we obtain

$$
\Delta z\left(t_{1}, x\right)=\int_{t_{0}}^{t_{1}} \int_{x_{0}}^{x} \Delta z_{\tau s}(\tau, s) d s d \tau
$$

Therefore,

$$
\begin{gathered}
\int_{t_{1}}^{t_{2}} \int_{x_{0}}^{t_{1}} \psi_{2}^{0^{\prime}}(t, x) E(x) \Delta z\left(t_{1}, x\right) d x d t= \\
=\int_{t_{0}}^{t_{1}} \int_{x_{0}}^{x_{1}}\left[\int_{t_{1}}^{t_{2}} \int_{x}^{x_{1}} \psi_{2}^{0^{\prime}}(\tau, s) E(s) \Delta z_{t x}(t, x) d s d \tau\right] d x d t .
\end{gathered}
$$

Identities (14)-(19) allow increment (17) of the quality criterion to be written in the form

$$
\begin{gathered}
\Delta S\left(u^{0}, v^{0}\right)=\int_{t_{0}}^{t_{1}} \int_{x_{0}}^{x_{1}} c^{\prime} \Delta z_{t x}(t, x) d t d x+d^{\prime} \Delta y\left(t_{2}, x_{1}\right)+ \\
=\int_{t_{0}}^{t_{1}} \int_{x_{0}}^{x_{1}} \psi_{1}^{0^{\prime}}(t, x) \Delta z_{t x}(t, x) d x d t-\int_{t_{0}}^{x_{1}} \int_{x_{0}}^{t_{2}}\left(\int_{t}^{x_{1}} \int_{x}^{t_{1}} \psi_{1}^{0^{\prime}}(\tau, s) A(\tau, s) d s d \tau\right) \Delta z_{t x}(t, x) d x d t- \\
-\int_{t_{0}}^{t_{1}} \int_{x_{0}}^{x_{1}}\left(\int_{x}^{x_{1}} \psi_{1}^{x_{1}^{\prime}}(t, s) B(t, s) d s\right) \Delta z_{t x}(t, x) d x d t- \\
-\int_{t_{0}}^{t_{1}} \int_{x_{0}}^{t_{1}}\left(\int_{t}^{t_{t}} \psi_{1}^{0^{\prime}}(\tau, x) C(\tau, x) d \tau\right) \Delta z_{t x}(t, x) d x d t-
\end{gathered}
$$




$$
\begin{gathered}
-\int_{t_{0}}^{t_{1}} \int_{x_{0}}^{x_{1}} \psi_{1}^{0^{\prime}}(t, x)\left(f(t, x, \bar{u}(t, x))-f\left(t, x, u^{0}(t, x)\right)\right) d x d t+ \\
+\int_{t_{1}}^{t_{2}} \int_{x_{0}}^{x_{1}} \psi_{2}^{0^{\prime}}(t, x) \Delta y(t, x) d x d t-\int_{t_{1}}^{t_{2}} \int_{x_{0}}^{x_{1}}\left[\int_{t}^{t_{2}} \int_{x}^{x_{1}} \psi_{2}^{0^{\prime}}(\tau, s) D(\tau, s, t, x)\right] \Delta y(t, x) d x d t- \\
-\int_{t_{1}}^{t_{x_{0}}} \int_{t}^{t_{2}}\left[\int_{x}^{x_{1}} \int_{2}^{0^{\prime}}(\tau, s)\left(g(\tau, s, t, x, \bar{v}(t, x))-g\left(\tau, s, t, x, v^{0}(t, x)\right)\right) d s d \tau\right] d x d t- \\
-\int_{t_{0}}^{t_{1}} \int_{x_{0}}^{x_{1}}\left[\int_{t_{1}}^{t_{2}} \int_{x}^{x_{1}} \psi_{2}^{0^{\prime}}(\tau, s) E(s) d s d \tau\right] \Delta z_{t x}(t, x) d x d t .
\end{gathered}
$$

Further, it follows from (10) that

$$
\begin{gathered}
\Delta y\left(t_{2}, x_{1}\right)= \\
=\int_{t_{1}}^{t_{2}} \int_{x_{0}}^{x_{1}}\left[D\left(t_{2}, x_{1}, t, x\right) \Delta y(t, x)+g\left(t_{2}, x_{1}, t, x, \bar{v}(t, x)\right)-g\left(t_{2}, x_{1}, t, x, v^{0}(t, x)\right)\right] d x d t+ \\
+\int_{t_{0}}^{t_{1}} \int_{x_{0}}^{x_{1}} E(x) \Delta z_{t x}(t, x) d x d t .
\end{gathered}
$$

Then increment formula (20) takes the form

$$
\begin{aligned}
& \Delta S\left(u^{0}, v^{0}\right)=\int_{t_{0}}^{t_{1}} \int_{x_{0}}^{x_{1}} c^{\prime} \Delta z_{t x}(t, x) d t d x+ \\
& +\int_{t_{0}}^{t_{1}} \int_{x_{0}}^{x_{1}} \psi_{1}^{0^{\prime}}(t, x) \Delta z_{t x}(t, x) d x d t-\int_{t_{0}}^{t_{1}} \int_{x_{0}}^{x_{1}}\left(\int_{t}^{t_{2}} \int_{x}^{x_{1}} \psi_{1}^{0^{\prime}}(\tau, s) A(\tau, s) d s d \tau\right) \Delta z_{t x}(t, x) d x d t- \\
& -\int_{t_{0}}^{t_{1}} \int_{x_{0}}^{x_{1}}\left(\int_{x}^{x_{1}} \psi_{1}^{0^{\prime}}(t, s) B(t, s) d s\right) \Delta z_{t x}(t, x) d x d t- \\
& -\int_{t_{0}}^{t_{1}} \int_{x_{0}}^{x_{1}}\left(\int_{t}^{t_{1}} \psi_{1}^{0^{\prime}}(\tau, x) C(\tau, x) d \tau\right) \Delta z_{t x}(t, x) d x d t- \\
& -\int_{t_{0}}^{t_{1}} \int_{x_{0}}^{x_{1}} \psi_{1}^{0^{\prime}}(t, x)\left(f(t, x, \bar{u}(t, x))-f\left(t, x, u^{0}(t, x)\right)\right) d x d t+ \\
& +\int_{t_{1}}^{t_{2}} \int_{x_{0}}^{x_{1}} \psi_{2}^{0^{\prime}}(t, x) \Delta y(t, x) d x d t-\int_{t_{0}}^{t_{1}} \int_{x_{0}}^{x_{1}}\left[\int_{t}^{t_{2}} \int_{x}^{x_{1}} \psi_{2}^{0^{\prime}}(\tau, s) D(\tau, s, t, x)\right] \Delta y(t, x) d x d t- \\
& -\int_{t_{1}}^{t_{2}} \int_{x_{0}}^{x_{1}}\left[\int_{t}^{t_{2}} \int_{x}^{x_{1}} \psi_{2}^{0^{\prime}}(\tau, s)\left(g(\tau, s, t, x, \bar{v}(t, x))-g\left(\tau, s, t, x, v^{0}(t, x)\right)\right) d s d \tau\right] d x d t-
\end{aligned}
$$




$$
\begin{gathered}
-\int_{t_{0}}^{t_{1}} \int_{x_{0}}^{x_{1}}\left[\int_{t_{1}}^{t_{2}} \int_{x}^{x_{1}} \psi_{2}^{0^{\prime}}(\tau, s) E(s) d s d \tau\right] \Delta z_{t x}(t, x) d x d t+ \\
+\int_{t_{1}}^{t_{2}} \int_{x_{0}}^{x_{1}} d^{\prime} D\left(t_{2}, x_{1}, t, x\right) \Delta y(t, x) d x d t+\int_{t_{0}}^{t_{1}} \int_{x_{0}}^{x_{1}} d^{\prime} E(x) \Delta z_{t x}(t, x) d x d t+ \\
\int_{t_{1}}^{t_{2}} \int_{x_{0}}^{x_{1}} d^{\prime}\left[g\left(t_{2}, x_{1}, t, x, \bar{v}(t, x)\right)-g\left(t_{2}, x_{1}, t, x, v^{0}(t, x)\right)\right] d x d t .
\end{gathered}
$$

Let $\psi_{1}^{0}(t, x), \psi_{2}^{0}(t, x)$ satisfy the relations (a conjugate system)

$$
\begin{gathered}
\psi_{1}^{0}(t, x)=-c+\int_{t}^{t_{2}} \int_{x}^{x_{1}} \psi_{1}^{0^{\prime}}(\tau, s) A(\tau, s) d s d \tau+\int_{x}^{x_{1}} B^{\prime}(t, s) \psi_{1}^{0}(t, s) d s+ \\
+\int_{t}^{t_{1}} C^{\prime}(\tau, x) \psi_{1}^{0}(\tau, x) d \tau-E^{\prime}(x) d+\int_{t_{1}}^{t_{2}} \int_{x_{0}}^{x_{1}} E^{\prime}(s) \psi_{2}^{0}(\tau, s) d s d \tau \\
\psi_{2}^{0}(t, x)=-D^{\prime}\left(t_{2}, x_{1}, t, x\right) d+\int_{t}^{t_{1}} \int_{x}^{x_{1}} D^{\prime}(\tau, s, t, x) \psi_{2}^{0}(\tau, s) d s d \tau .
\end{gathered}
$$

Then increment formula (21) takes the form

$$
\begin{aligned}
& \Delta S\left(u^{0}, v^{0}\right)=-\int_{t_{0}}^{t_{1}} \int_{x_{0}}^{x_{1}} \psi_{1}^{0^{\prime}}(t, x)\left(f(t, x, \bar{u}(t, x))-f\left(t, x, u^{0}(t, x)\right)\right) d x d t- \\
& -\int_{t_{1}}^{t_{2}} \int_{x_{0}}^{x_{1}}\left[\int_{t}^{t_{2}} \int_{x}^{x_{1}} \psi_{2}^{0^{\prime}}(\tau, s)\left(g(\tau, s, t, x, \bar{v}(t, x))-g\left(\tau, s, t, x, v^{0}(t, x)\right)\right) d s d \tau\right] d x d t .
\end{aligned}
$$

Introducing the notation

$$
\begin{gathered}
H_{1}\left(t, x, u, \psi_{1}^{0}\right)=\psi_{1}^{0^{\prime}} f(t, x, u), \\
H_{2}\left(t, x, v, \psi_{2}^{0}\right)=\int_{t}^{t_{2}} \int_{x}^{x_{1}} \psi_{2}^{0^{\prime}}(\tau, s) g(\tau, s, t, x, v(t, x)) d s d \tau-d^{\prime} g\left(t_{2}, x_{1}, t, x, v(t, x)\right) .
\end{gathered}
$$

we write formula (24) in the following form:

$$
\begin{gathered}
\Delta S\left(u^{0}, v^{0}\right)=-\int_{t_{0}}^{t_{1}} \int_{x_{0}}^{x_{1}}\left(H_{1}\left(t, x, \bar{u}(t, x), \psi_{1}^{0}(t, x)\right)-H_{1}\left(t, x, u^{0}(t, x), \psi_{1}^{0}(t, x)\right)\right) d x d t- \\
-\int_{t_{1}}^{t_{2}} \int_{x_{0}}^{x_{1}}\left(H_{2}\left(\tau, s, t, x, \bar{v}(t, x), \psi_{2}^{0}(t, x)\right)-H_{2}\left(\tau, s, t, x, v^{0}(t, x), \psi_{2}^{0}(t, x)\right)\right) d x d t .
\end{gathered}
$$

From here, using an analogue of McShane's needle-shaped variation (see, e.g., [1,9]), we can prove

Theorem. The necessary and sufficient condition of the optimality of the admissible control $\left(u^{0}(t, x), v^{0}(t, x)\right)$ in the problem under investigation is that the relation 


$$
\max _{u \in U} H_{1}\left(\theta, \xi, u, \psi_{1}^{0}(\theta, \xi)\right)=H_{1}\left(\theta, \xi, u^{0}(\theta, \xi), \psi_{1}^{0}(\theta, \xi)\right),
$$

should be true for all $(\theta, \xi) \in\left[t_{0}, t_{1}\right) \times\left[x_{0}, x_{1}\right)$, and the relation

$$
\max _{v \in V} H_{2}\left(\alpha, \beta, v, \psi_{2}^{0}(\alpha, \beta)\right)=H_{2}\left(\alpha, \beta, v^{0}(\theta, \xi), \psi_{2}^{0}(\alpha, \beta)\right),
$$

for all $(\alpha, \beta) \in\left[t_{1}, t_{2}\right) \times\left[x_{0}, x_{1}\right)$.

Here, $(\theta, \xi) \in\left[t_{0}, t_{1}\right) \times\left[x_{0}, x_{1}\right)$ and $(\alpha, \beta) \in\left[t_{1}, t_{2}\right) \times\left[x_{0}, x_{1}\right)$ are regular points (Lebesgue points [7,9]) of the controls $u^{0}(t, x)$ and $v^{0}(t, x)$, respectively.

Note that in the case of a nonlinear but convex quality criterion similarly, one can prove a sufficient optimality condition of the type of L.S. Pontryagin's maximum principle.

\section{Conclusion}

In the problem under consideration, a conjugate system was introduced for the considered unconventional control problem, using its specific features. This made it possible, using the constructed formula for the increment of the quality functional, to prove a necessary and sufficient optimality condition of the Pontryagin maximum principle type.

\section{References}

[1] К.Б. Мансимов, М.Дж. Марданов, Качественная теория оптимального управления системами Гурса-Дарбу, Баку, Изд-во ЭЛМ, (2010) 360 р. [In Russian: K.В. Mansimov, M.J. Mardanov, Qualitative Theory of Optimal Control of Goursat-Darboux Systems, Baku, ELM].

[2] А.А. Абдуллаев, К.Б. Мансимов, Необходимые условия оптимальности в процессах, описываемых системой интегральных уравнений типа Вольтерра, Баку, ЭЛМ, (2013) 224 p. [In Russian: A.A. Abdullayev, K.B. Mansimov, Necessary optimality conditions in processes described by a system of Volterra integral equations, Baku, ELM].

[3] К.Б. Мансимов, Особые управления в задачах управления системами с распределенными параметрами, Современная математика и ее приложения. 42 (2006) pp.39-83. [In Russian: K.B. Mansimov, Special controls in problems of control of systems with distributed parameters, Sovremennaya matematika i yeye prilozheniya].

[4] В.И. Плотников, В.И. Сумин, Проблема устойчивости нелинейных систем Гурса-Дарбу, Дифференц. урав. 5 (1972) pp.845-856. [In Russian: V.I. Plotnikov, V.I. Sumin, The stability problem for nonlinear GoursatDarboux systems, Differ. urav.].

[5] К. К. Гасанов, О существовании оптимальных управлений для процессов, описываемых системой гиперболических уравнений, Ж. вычисл. матем. и матем. физ. 13:3 (1973) pp.599-608 [In Russian: K.K. Hasanov, On the existence of optimal controls for processes described by a system of hyperbolic equations, Zh. vychisl. mat. and matem. fiz.].

[6] З.Б. Цалюк, Интегральные уравнения Вольтерра, Итоги науки и техники, сер. Математический анализ. 15 (1977), pp.131-198. [In Russian: Z.B. Tsalyuk, Volterra Integral Equations, Results of science and technology, Matematicheskiy analiz series].

[7] М.М. Новоженов, В.И. Сумин, М.М. Сумин, Методы оптимального управления системами математической физики, Горький, ГГУ, (1986) 87 p. [In Russian: M.M. Novozhenov, V.I. Sumin, M.M. Sumin, Methods of optimal control of mathematical physics systems, Gorky, GSU].

[8] В.М. Алексеев, В.М. Тихомиров, С.В. Фомин, Оптимальное управление, Москва, Физматлит, (2005) 429 р. [In Russian: V.M. Alekseyev, V.M. Tikhomirov, S.V. Fomin, Optimal control, Moscow, Fizmatlit].

[9] Л.С. Понтрягин, В.Г. Болтянский, Р.В. Гамкрелидзе, Е.Ф. Мищенко, Математическая теория оптимальных процессов, Москва, Наука, (1984) 386 р. [In Russian: L.S. Pontryagin, V.G. Boltyansky, R.V. Gamkrelidze, E.F. Mishchenko, Mathematical theory of optimal processes, Moscow, Nauka]. 